\title{
Metallicity and kinematical clues to the formation of the Local Group
}

\author{
Rosemary F. G. Wyse ${ }^{1, \star}$ \\ ${ }^{1}$ Johns Hopkins University, Department of Physics \& Astronomy, Baltimore, MD 21218, USA
}

Received 30 May 2005, accepted 11 Nov 2005

Published online later

Key words dark matter - galaxies: formation - galaxies: kinematics and dynamics - Local Group - Galaxy: formation - Galaxy: stellar content - stars: abundances

\begin{abstract}
The kinematics and elemental abundances of resolved stars in the nearby Universe can be used to infer conditions at high redshift, trace how galaxies evolve and constrain the nature of dark matter. This approach is complementary to direct study of systems at high redshift, but I will show that analysis of individual stars allows one to break degeneracies, such as between star formation rate and stellar Initial Mass Function, that complicate the analysis of unresolved, distant galaxies.
\end{abstract}

(C) 2010 WILEY-VCH Verlag GmbH \& Co. KGaA, Weinheim

\section{Introduction}

These are exciting times to study local galaxies, due to a convergence of capabilities:

- Large observational surveys of individual stars in Local Group galaxies are feasible, using wide-field imagers and multi-object spectroscopy, complemented by spacebased imaging and spectroscopy, with in the near-future the astrometric satellite Gaia providing 6-dimensional phase-space information

$\rightarrow$ Imaging surveys need matched spectroscopic surveys for full physics

- High-redshift surveys are now starting to quantify the stellar populations and morphologies of galaxies at high look-back times, equal to the ages of old stars nearby

- Large, high-resolution simulations of structure formation are allowing predictions of Galaxy formation in a cosmological context

I will focus on the first of these capabilities, which provides the data that enables the fast-growing field of Galactic Archaeology. In this approach, analysis of the properties of low-mass old stars in our own Galaxy, and in nearby galaxies, allows us to do cosmology, locally. There are copious numbers of stars nearby that have ages greater than $\sim 10$ Gyr: these formed at look-back times corresponding to redshifts $>2$, and for a subset, perhaps as early as the epoch of reionization. The 'fossil record' is comprised of the conserved quantities that reveal conditions at the time the star formed, such as the chemical abundances in the interstellar medium. This is a complementary approach to direct study of galaxies at high redshift; the two approaches to understanding galaxy evolution analyse on the one hand snapshots of different galaxies at different times (redshifts)

\footnotetext{
^ Corresponding author: e-mail: wyse@pha.jhu.edu
}

and on the other hand the evolution of one galaxy. A significant advantage of Galactic Archaeology, studying resolved stars, is that one can derive separately the stellar metallicity distributions and age distributions, and constrain variations in the massive-star Initial Mass Function (IMF) from elemental ratios. One can then break degeneracies that hamper the interpretation of photometry and even spectra of the integrated light of galaxies, including the well-known age-metallicity case and star-formation-rate-IMF. Analysis of the motions of stars within a given galaxy can also provide the mass profile of the underlying potential, going from kinematics to dynamics.

The key questions that can be addressed, and which I will touch on in this review, include

- How do galaxies form?

$\rightarrow$ star formation histories

$\rightarrow$ mass assembly histories

$\rightarrow$ link to black hole growth

$\rightarrow$ the physics of 'feedback'

- What is the nature of dark matter?

$\rightarrow$ determines potential well shape

$\rightarrow$ determines merger histories

\section{Testing the $\Lambda$ CDM paradigm of structure formation}

The standard model of structure formation is that of $\Lambda \mathrm{CDM}$, where most of the gravitating material in the Universe is in the form of cold dark matter, and most of the energy is in a dark component that behaves as a constant density Cosmological Constant. This has proven to be an excellent description of the Universe on large scales, probed by the Cosmic Microwave background (e.g. Komatsu et al. 2009), and the large-scale power-spectrum of the distribution of galaxies (e.g. Percival et al. 2010). Gravity dominates on these 
scales, so that hot and cold dark matter fit equally well, and the physics of the constituents of dark matter is expected to be manifest only on smaller scales, such as sparse galaxy groups and individual galaxies (e.g. Ostriker \& Steinhardt 2003). These are indeed the scales on which cracks are appearing in the $\Lambda$ CDM armour (e.g. Wyse 2001; Peebles \& Nusser 2010).

Structure formation in the $\Lambda \mathrm{CDM}$ paradigm is hierarchical, with smaller scales forming first, due to the shape of the power spectrum of primordial density fluctations. This results in galaxy formation and evolution being largely driven by mergers. The outcome of a merger between two galaxies (i.e. dark matter haloes with associated baryonic material bound within them) depends in large part to the relative fractions of dissipationless material (dark matter, stars) and dissipational material (gas - ignoring dissipational dark matter models) (e.g. Tinsley \& Larson 1979; Zurek, Quinn \& Salmon 1988; Governato et al. 2009), the assumed physical conditions and equation of state of the dissipational material. The mass ratio and relative densities of the merging systems are also extremely important. Simulations that are dark-matter-only have the simplest physics, and - following on from the pioneering work of Moore et al. (1999) and of Klypin et al. (1999) - the highest resolution realizations for a Milky Way galaxy analogue show significant merging at all redshifts, and persistent substructure on all mass scales throughout the final, zero redshift, galaxy (e.g. Stadel et al. 2009). Substructure makes its presence felt through its gravitational interactions and assimilation through mergers, which can lead to heating of initially cold stellar components (e.g. Quinn \& Goodman 1986; Kazantzidis et al. 2009). Thin disks are very susceptible to the destructive effects of mergers (of roughly equal mass), and in the $\Lambda \mathrm{CDM}$ scenario the disks we see today largely formed after merging ceases to be active, which is typically a redshift $z \sim 1$. The possibility that gas accretion into dark halos is dominated by cold streams, rather than the steady accretion of gas initially shock-heated to the halo virial temperature (White \& Rees 1978), leads to the creation of fat, clumpy, turbulent disks of gas and stars at higher redshifts, $z \sim 2$ with active star formation (Ceverino, Dekel \& Bournaud 2010), evolving into quiescent spheroid-dominated systems at lower redshift. The initial formation at $z \sim 2$ of thin disks - that are later destroyed through merging - is seen in high-resolution multi-phase hydrodynamic simulations within the $\Lambda \mathrm{CDM}$ framework (e.g. Scannapieco et al. 2009). The relationship of these predicted early thick and thin disks to the starforming galaxies observed at redshifts $\sim 2$ (e.g. Genzel 2009) is as yet unclear, but intriguing.

A thin stellar disk is likely to be significantly affected by even very unequal mass-ratio mergers (minor mergers), with a significant fraction of the pre-merger orbital energy being absorbed into the internal degrees of freedom of the disk, resulting in heating, and creation of a thick stellar disk. Gas of course can radiate energy and cool, but the stellar component of the disk cannot. Adiabatic compression of the heated stellar disk,by gas settling later into the midplane,results in both a decrease of scale height of the stellar component and an increase of its velocity dispersion (e.g. Toth \& Ostriker 1990; Elmegreen \& Elmegreen 2006). Some of the orbital angular momentum will also be absorbed, in general resulting in a tilt of the disk plane. The relative density profiles and the details of the satellite's orbital parameters also are important determinants of the final outcome, in terms of the stellar disk structure (and of the fate of the satellite).

The robustness, masses and orbital parameters of substructure in $\Lambda \mathrm{CDM}$, and the typical merging history of large galaxies, leads to the expectation that thick disks should be extremely common, and that the heating of thin disks should continue to late times. A vivid illustration of the results, using a pure N-body code and a cosmologically motivated retinue of satellite galaxies impinging on a stellar thin disk, assumed in place at redshift $z=1$, are shown in Kazantzidis et al. (2009). As noted in several papers, in the concordance $\Lambda \mathrm{CDM}$ cosmology, a typical dark halo of the mass of that inferred for the Milky Way $\left(\sim 10^{12} \mathrm{M}_{\odot}\right)$ will have suffered significant merging with relatively massive satellites (up to $10^{11} \mathrm{M}_{\odot}$ ) since a redshift of unity (e.g. Stewart et al. 2008; Fakhouri, Ma \& Boylan-Kolchin, 2010). While the presence of gas may prevent the destruction of an existing thin disk, the heating is unavoidable, leaving an imprint in the stellar velocity dispersions (which as noted above, only increase with subsequent adiabatic growth of an embedded gas-rich thin disk).

Material is stripped from satellites as they merge, depending to first order on the relative density compared to that of the larger galaxy interior to the satellite orbit. The less dense, outer parts of satellites are thus stripped more easily, at larger distances from the central regions of the large galaxy. The typical orbits of merging substructure are elliptical, albeit with the eccentricity distribution peaking at close-to-parabolic orbits (e.g. Benson 2005; Khochfar \& Burkert 2006). Debris from a given satellite that survives to the disk plane is broadly expected to be distributed in a thick torus, with radial extent indicating the location at which the satellite was disrupted, and some stars in the debris could have orbital parameters similar to the heated thin disk, now the thick disk (e.g. Huang \& Carlberg 1997), and even to the surviving old thin disk (Abadi et al. 2003). The dark matter stripped from satellites during merging also builds up a dark-matter disk (Read et al. 09), the mass of which is severely constrained, at least locally, by the vertical motions of stars, which are consistent with no significant disk dark matter (the ' $K_{Z}$ ' analysis and Oort limit, Kuijken \& Gilmore 1989, 1991; Holmberg \& Flynn 2004; Bienaymé et al. 2006). Torques during a minor merger will drive gas and stars inwards, to contribute to the inner disk and bulge (e.g. Mihos \& Hernquist 1996).

In $\Lambda \mathrm{CDM}$, the stellar halo of disk galaxies is postulated to be formed from disrupted satellite galaxies, with structure in coordinate space persisting for many dynamical 
times, even to the present times in the outer regions, where timescales are longest. Graphic illustration of the predicted structure, should the stellar halo consist entirely of accreted satellites, is given in Johnston et al. (2008; note that this is not a fully self-consistent model). Some fraction of the stars now in the inner halo/bulge may be disrupted disks from earlier stages of sub-halo merging, giving a 'dual halo' (e.g. Zolotov et al. 09).

\subsection{The Milky Way and M31 as templates}

As noted above, the stellar halo, bulge, thick disk and even some part of (old?) thin disk of a typical large disk galaxy is predicted, in $\Lambda \mathrm{CDM}$, to be created through the effects of mergers. One should see signatures of these origins in the stellar populations, since some memory of the initial conditions when a star is formed is retained. The Local Group provides an ideal test-bed of such predictions. Observational effects include

- Coordinate space structure

- Kinematic (sub)structure

- Chemical abundance signatures

- Distinct age distributions

- Properties of surviving satellite galaxies

Kinematic phase-space structure should survive longer than will coordinate-space structure, and chemical signatures of distinct populations are the most long-lived. Kinematics and chemical distributions are most robustly determined through spectroscopy.

Stars today probe conditions at a look-back time equal to their age; 10Gyr corresponds to a redshift $z \sim 2$ for the concordance $\Lambda \mathrm{CDM}$ cosmology. Such stars, represented by main sequence, red giant stars and horizontal branch stars, are accessible in the Local Group with current capabilities. Only young, massive stars may be studied currently in galaxies beyond the Local Group; the wealth of information that can be derived from these was described by Rolf Kudritzki in his Schwarzschild lecture; an Extremely Large telescope is required to reach lower mass stars beyond the Local Group.

The Local Group consists of two large disk galaxies, M31 and the Milky Way galaxy, plus a low luminosity, very late-type disk galaxy, M33, a companion to M31. There are also retinues of gas-rich dwarf irregular (dIrr) and gaspoor dwarf spheroidal (dSph) satellite galaxies of each of the large disk galaxies, with many recent discoveries (discussed further below). We will discuss how photometric and (in particular) spectroscopic surveys of these galaxies reveal the important processes by which galaxies evolve.

\section{Photometric surveys}

\subsection{The Milky Way galaxy}

The first irrefutable evidence for ongoing merging between the Milky Way and its satellite galaxies was provided by

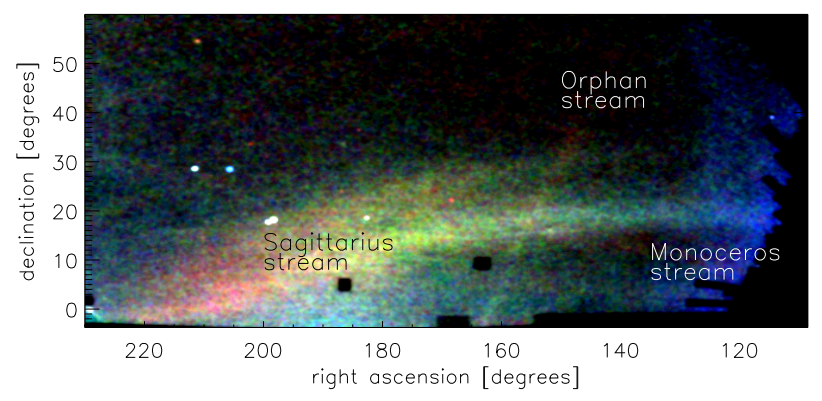

Fig. 1 The 'Field of Streams' - and dots. This annotated version is courtesy of Gerry Gilmore and Vasily Belokurov (see Belokurov et al. 2006). The figure shows the distribution of main sequence turnoff stars on the sky, selected from the SDSS imaging data by the colour and magnitude cuts $19.0<r<22.0$ and $g-r<0.4$, colour-coded in the figure by apparent magnitude, i.e. by distance from the Sun. Blue represents the nearest stars (at $\sim 10 \mathrm{kpc}$ ), then green, then red (at $\sim 30 \mathrm{kpc}$ ). The distribution of stars is clearly not uniform or smooth.

the (serendipitous) discovery of the Sagittarius ( $\mathrm{Sgr}$ ) dSph galaxy, by Ibata, Gilmore \& Irwin (1994). The coordinatespace overdensity of the core of this galaxy is illustrated in Fig. 1 of Wyse, Gilmore \& Franx (1997). This galaxy was detected kinematically during a spectroscopic study of the bulge of the Milky Way (Ibata \& Gilmore 1995). Member stars of this 'moving group' also have a redder colour distribution than the field bulge stars, a manifestation of their distinct age and metallicity distributions (determined by subsequent observations e.g. Layden \& Sarajedini 2000; McWilliam \& Smecker-Hane 2005; Sbordone et al. 2007; Siegel et al. 2007; Chou et al. 2010). The Sgr dSph contains no detectable gas, down to limits of $\sim 140 \mathrm{M}_{\odot}$ of atomic Hydrogen (Grcevich \& Putman 2009 and refs. therein), and is clearly dark-matter dominated, based on the velocity dispersion of its member stars (e.g. Ibata et al. 1997). This lack of gas and dominance by dark matter are characteristic of the dSph satellite galaxies.

A new vitality was injected into the field by the advent of the Sloan Digital Sky Survey (SDSS) imaging data. The uniformly excellent photometry across a large fraction of the Northern sky revealed a very non-uniform distribution of resolved stars, dubbed the 'Field of Streams' (Belokurov et al. 2006; see Fig. 1 here).

The distribution of stars in the Northern sky shown in Fig. 1 is dominated by the two swathes of stars in the tidal streams from the Sgr dSph. Tidal debris from this system appears to be manifest not only in these two wraps (e.g. Fellhauer et al. 2006) but also as a significant part of the Virgo overdensity (e.g. Prior, Da Costa \& Keller 2009). The outer stellar halo, at Galactocentric distances of greater than $15 \mathrm{kpc}$ and traced out to $\sim 60 \mathrm{kpc}$, shows significant coordinate space structure (Bell et al. 2008; Watkins et al. 2009), the vast majority of which is associated with the Sgr dSph, and to a lesser extent with the Hercules-Aquila Cloud (Be- 


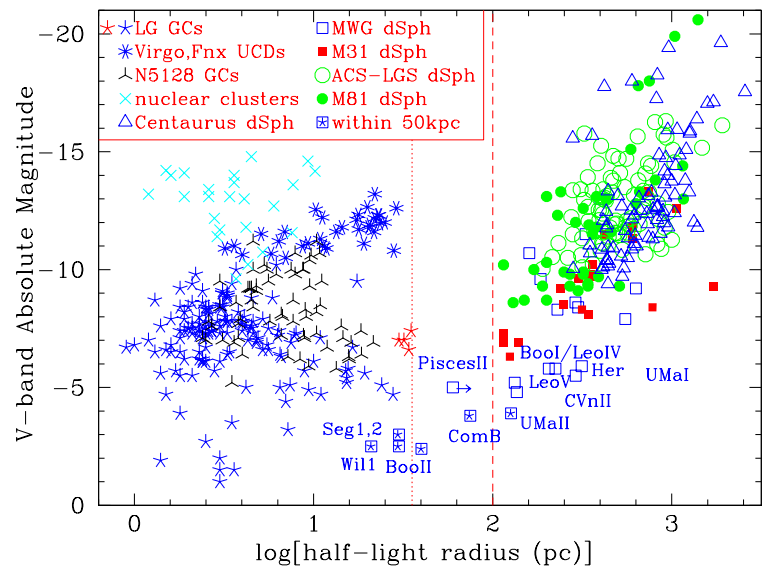

Fig. 2 Distribution of star clusters and dwarf galaxies in the plane of stellar half-light radius $v s$ absolute V-band magnitude. The faint systems discovered in the SDSS imaging data that have been reported to be galaxies are identified by name. There are only three systems between the vertical red lines (dotted on the left, at a half-light radius of $36 \mathrm{pc}$, and dashed on the right, at a half-light radius of $100 \mathrm{pc}$ ).

lokurov et al. 2007a), accounting for perhaps $10 \%$ of the total mass of the stellar halo $\left(\mathbf{M}_{\text {star, halo }} \sim 10^{9} \mathbf{M}_{\odot}\right)$.

The numerous 'dots' are also of extreme interest: these are gravitationally bound satellites of the Milky Way, ranging in luminosity from only a few hundred solar luminosities to millions of solar luminosities. Whether they are star clusters or satellite galaxies is only revealed by spectroscopic measurements of their internal kinematics, from radial velocities of member stars - galaxies, by definition, are held together by the gravity of dark matter haloes, while star clusters are baryon-dominated self-gravitating systems; this is discussed further below. Photometry alone provides distances - from the colour-magnitude diagram (CMD) of member stars - the scale-lengths of the stellar distribution, and the total luminosity. These result in the size-absolute magnitude plot shown in Fig. 2, which includes known star clusters and dwarf galaxies (and is an annotated update of Fig. 1 in Gilmore et al. 2007).

The unoccupied region at the lower right is a selection effect - too few stars over too large an area - and the boundary approximately follows a line of constant V-band surface brightness equal to $\sim 31 \mathrm{mag} / \mathrm{sq}$ arcsec (see Belokurov et al. 2007b). There is a noticeable gap, emphasised in the Figure by the vertical red lines. As the SDSS imaging data have allowed lower and lower luminous systems to be discovered, they have largely been on either side of the gap (the newly discovered faint globular clusters are unlabelled). Indeed only three systems fall in the gap. Of these, two are closer than $50 \mathrm{kpc}$, within the realm of expected tidal effects - manifest in the Sgr streams - and one, Pisces II, is surrounded by a 'sea' of blue horizontal branch stars that were not included in the estimation of the half-light radius (Belokurov et al. 2010), and so will likely move out of the gap, to larger sizes, with deeper photometry. The nearby systems, within $50 \mathrm{kpc}$, are indicated in Figure 2 and these tend to be rather elliptical and/or distorted (e.g. Martin, de Jong \& Rix 2008), which may well indicate the effects of tides. Their present structure cannot be assumed to be the equilibrium structure at formation.

\subsection{M31}

This subsection should perhaps be labelled 'M31 / M33', since deep imaging surveys are revealing hints of low surface brightness stellar features between the two, which, together with the wide-spread HI gas (Putman et al. 2009) are suggestive of past interactions. The most comprehensive wide-field survey to date is that of the PAndAS collaboration (McConnachie et al. 2009), building upon the pioneering work of Ibata et al. (2001) and Ferguson et al. (2002). Evolved stars in either of the hydrogen-shell burning phase (red giants) or core-helium burning phase (red clump and horizontal branch) are fairly straightforward to image at the distance of M31, at least in the lower surface-brightness outer disk and halo (avoiding source crowding and confusion). Similarly to the dominant stream from the Sgr dSph in the Milky Way, the structure in the stellar halo of M31 is dominated by the 'Giant Stream' (Ibata et al. 2004), together with significant other structure (e.g. Tanaka et al. 2010). Not only the Giant Stream but also smaller-scale structure can result from one accreted system (e.g. Fardal et al. 2008), but as in the Milky Way the situation is complex and many merged satellites may be involved (Font et al. 2008).

Again, many new satellites of M31 have been discovered by deep imaging surveys (e.g. Martin et al. 2009 and references therein); these are included in the satellite systems shown in Fig. 2, the M31 satellites also avoid the size gap. One might note that the earlier finding of significantly larger radii of M31 satellites compared to those of the Milky Way (McConnachie \& Irwn 2006) is not held up by the fainter systems.

The colour of a red giant star of a given luminosity is more sensitive to chemical abundance than to age. Counts of red giants divided into two categories, red and blue, reveal significant chemical inhomogeneities across the outer disk and halo of M31 (Ferguson et al. 2002). The mean metallicity of the dominant 'halo' (really 'non-thin-disk') component of M31, derived from colours of the RGB stars, is significantly higher than that of the stellar halo of the Milky Way, being $[\mathrm{Fe} / \mathrm{H}] \sim-0.6$ dex (cf. Mould \& Kristian 1986; Durrell, Harris \& Pritchet 2004). This is equal to the mean metallicity of the Galactic thick disk at the solar neighbourhood, leading to speculation that these stars represent the thick disk of M31, and that the Galactic thick disk, rather than the halo, should be called 'Population II' to match the population in M31 that Baade resolved into stars (Wyse \& Gilmore 1988). This high a value of mean chemical abundance, combined with the inferred old age from the CMD, 
implies that the stars formed within a fairly deep potential well, significantly more massive than a typical dwarf galaxy.

A large, high $\mathrm{S} / \mathrm{N}$ spectroscopic survey is required to provide the kinematics and robust metallicities of both the field stars and satellite galaxies. This will allow testing of models for interactions between M31 and its satellites, including M33, and masses estimates for the surviving satellites. These have been initiated with $8 \mathrm{~m}$-class telescopes (e.g. Chapman et al. 2008; Letarte et al. 2009; Gilbert et al. 2009; Kalirai et al. 2010).

\section{Spectroscopic surveys}

While there exist photometric techniques, such as the colour of the RGB with an assumed age, or the UV excess of main sequence $F / G$ stars (measured either by broad-band filters or Strömgren photometry), to determine chemical abundances, and other photometric techniques for effective temperatures, spectroscopy remains the most robust and reliable means to determine stellar parameters, in particular the elemental abundances. Spectroscopy is also critical for determining kinematics for stars, particularly those far enough away that proper motions are too small to be measured (in the era prior to Gaia!)

There is a need for a variety of spectroscopic surveys at different spectral resolutions. Moderate resolution, here defined as a resolving power of a few thousand at optical wavelengths, provides spectra that are sufficient to determine line-of-sight motions to better than $10 \mathrm{~km} / \mathrm{s}$ and metallicities to $\sim 0.2$ dex. With statistically large samples of stars these data can then be used to define the distribution functions of the major stellar populations under study, and investigate decompositions into different components. An individual star can then, in a probabilistic manner, be assigned to a given component. A significant amount of physics lies in the detailed shape and tails of the distribution functions, and these require large samples with well-defined selection functions.

High resolution, here defined as a resolving power of several tens of thousand at optical wavelengths, provides spectra that are sufficient to determine line-of-sight motions to much better than $1 \mathrm{~km} / \mathrm{s}$ and individual elemental abundances to $\sim 0.05$ dex. This precision in the velocities allows the mapping of cold stellar substructure, such as tidal streams, and the internal kinematics of low luminosity satellite galaxies. Ideally one would have both high and moderate resolution spectra for stars drawn from the same parent sample, such as is possible with the RAVE spectroscopic survey (e.g. Steinmetz et al. 2006), as discussed below.

\subsection{Masses and mass profiles of dwarf galaxies}

Precise measurement, with echelle spectroscopy, of the lineof-sight velocities of member stars of dSph satellite galaxies was pioneered by Marc Aaronson, who derived a high mass-to-light ratio for the Draco dSph, based on velocities of three carbon stars. He obtained a lower limit to the central velocity dispersion of $6.5 \mathrm{~km} / \mathrm{s}$ (Aaronson 1983), perfectly consistent with the modern value of $9 \mathrm{~km} / \mathrm{s}$ (Walker et al. 2009). Mass-to-light ratios have been estimated for the systems in Fig. 2 leading to the conclusion that all systems to the right of the gap are embedded in dark matter haloes, i.e. are galaxies, while all equilibrium systems to the left of the gap are star clusters. This then provides the physical interpretation of the size distribution in terms of a minimum scale for systems dominated by dark matter (Gilmore et al. 2007). The scale in Fig. 2 is that of the light; given that baryons dissipate binding energy while gaseous, and become self-gravitating to form stars, this stellar half-light radius is expected to be a lower limit to the dark-matter scalelength. Such a minimum scale to dark matter of greater than $\sim 100 \mathrm{pc}$ is not expected if the dark matter is cold, and may be indicative of warm dark matter, such as sterile neutrinos (e.g. Kusenko 2009).

Derivation of mass profiles, as opposed to estimates of total mass, requires velocity information as a function of projected radius, not just for the central regions. The most straightforward analysis uses the Jeans equations, which relate the mass profile to the stellar light profile through the radial dependences of (line-of-sight) velocity dispersion and velocity dispersion anisotropy. However, this introduces a well-known mass-anisotropy degeneracy (Binney \& Mamon 1982), which can be broken by use of the detailed line-of-sight velocity distribution, rather than its moments, such as the velocity dispersion (Gerhard 1993; Gerhard et al. 1998). This latter approach requires sample sizes of thousands of stars, well-distributed across the face of the galaxy, but with the central regions remaining critical, since it is here that the physics of the dark matter particle will be manifest most strongly. Such datasets are now being acquired (Gilmore et al. in preparation). The spherical Jeans' equation approach, with assumed isotropy, has the advantage of simplicity and when applied to a set of galaxies can identify any differences among them. The results of such an investigation, applied to six of the classical dSph satellites of the Milky Way is shown in Fig. 3, taken from Gilmore et al. (2007) - the derived mass density profile for each galaxy favours an inner core, rather than the cusped profile predicted for cold dark matter (Navarro, Frenk \& White 1996). Further, all the profiles are very similar. This similarity is despite the fact that this set of galaxies covers a wide range in star-formation histories, implying that an appeal to feedback from star-formation to smooth out an inner cusp into a core would need to be tuned to each galaxy.

\subsection{Elemental abundances: beyond metallicity}

Different elements are created in stars of different masses and different evolutionary stages, on different timescales. Thus the pattern of elemental abundances seen in a stellar population reflects the star formation history and the stellar initial mass function (and the binary population) that provided the enrichment of the stars we observe now. A con- 


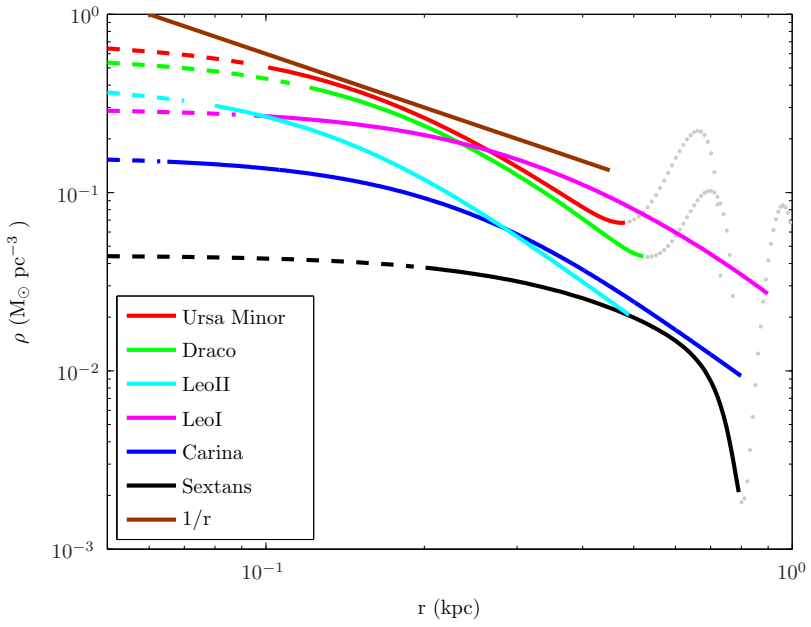

Fig. 3 Derived mass density profiles from (isotropic) Jeans-equation analyses of the stellar velocity dispersion profiles of 6 dwarf spheroidal galaxies. Also shown is a $r^{-1}$ profile, to which $C D M$-mass profiles are predicted to asymptote. The analysis is in each case reliable out to $r \sim 500$ pc. This figure is taken from Gilmore et al. (2007)

sequence is that old low-mass stars nearby provide insight into the stellar IMF at high redshift (look-back times at least equal to the age of the star).

Core-collapse supernovae, the death throes of massive, short-lived, stars, are the primary sources of the $\alpha$-elements, created in steady-state, pre-supernova nucleosynthesis and ejected in the explosion. There is little iron produced, since the iron core is photo-disintegrated and largely forms the stellar remnant. Type Ia supernovae are produced by explosive nucleosynthesis in a white dwarf of mass greater than the Chandrasekhar limit, created by accretion from a binary companion. Such systems evolve on timescales that can be as long as a Hubble time and provide mostly iron, about ten times as much per supernova as a Type II supernova.

Stars formed in the earliest stages of a self-enriching system will have high levels of $[\alpha / \mathrm{Fe}]$, reflecting the products of Type II (core-collapse) supernovae. There is negligible range in lifetime for Type II progenitors, compared to timescales of interest for star formation, and if there is good mixing of the supernova ejecta one expects to see an IMFaveraged yield of $[\alpha / \mathrm{Fe}]$ in the next generation of stars. The most massive Type II progenitors produce the highest values of [ $\alpha / \mathrm{Fe}]$ (Gibson 1998; Kobayashi et al. 2007), so that a massive-star IMF biased towards the most massive stars will leave a signature, namely even higher values of IMFaveraged $[\alpha / \mathrm{fe}]$. Some (model-dependent) time after the onset of star formation, white dwarfs will accrete sufficient material to explode as Type Ia supernovae. The first Type Ia are expected to result from progenitors with main sequence mass just below the threshold for core-collapse $\left(\sim 8 \mathrm{M}_{\odot}\right)$, and these could in principle evolve to explosion in less than $10^{8} \mathrm{yr}$ in the double-degenerate model (e.g. Matteucci et al. 2009). It takes longer for sufficient Type Ia to explode and for their ejecta to be incorporated in the next generation of stars, and a typical lag seen in chemical evolution models is $\sim 1$ Gyr (Matteucci et al. 2009). Of course, the iron abundance corresponding to this time depends on the star formation rate and gas flows in or out. Inefficient enrichment, due, for example, to a slow rate of star formation or to loss of metals by winds, results in the signature of 'extra' iron from Type Ia being seen at lower values of $[\mathrm{Fe} / \mathrm{H}]$. High rates of star formation and efficient enrichment will produce a 'Type II plateau' that extends to higher [Fe/H] (e.g. Fig. 1 of Wyse \& Gilmore 1993). One then expects that systems with low rates of star formation, over extended periods such as the dwarf spheroidal satellites of the Milky Way should show low values of $[\alpha / \mathrm{Fe}]$ at low values of $[\mathrm{Fe} / \mathrm{H}]$ (Unavane, Wyse \& Gilmore 1996).

Observations have confirmed this expectation. A compilation of $[\mathrm{Ca} / \mathrm{Fe}]$ for stars in the Milky Way and in representative gas-poor satellite galaxies is shown in Fig, 4, the stars in satellite galaxies lie systematically below the field halo stars at the same value of $[\mathrm{Fe} / \mathrm{H}]$, leading to the conclusion that accretion of stars from dwarf galaxies like those observed today could not play an important role in the formation of the stellar halo (Venn et al. 2004). Complementary, independent age information that the vast majority of halo stars are old, contrasting with the dominant intermediate age population of the luminous $\mathrm{dSph}$, further constrains progenitors to consist only of stars formed early, in a shortlived burst of star formation that happened a long time ago (e.g. Unavane et al. 1996). A progenitor that would have been expected to have an extended star formation history, similar to a typical $\mathrm{dSph}$, would have had to be accreted $\sim 10$ Gyr ago for its member stars to contribute to the bulk of the stellar halo (Unavane et al. 1996).

A new result is seen too, as observations have pushed to lower and lower metallicities within each system: we find, within the errors and limitations of small numbers, a consistent value for the enhanced 'Type II plateau' in $[\alpha / \mathrm{Fe}]$ in all galaxies for their lowest metallicity member stars. These are the stars which one might expect to have formed in the earliest stages of star formation within each individual galaxy, and thus to show (pre)enrichment by core-collapse supernovae only. The fact that there is only moderate scatter in $[\alpha / \mathrm{Fe}]$ in these stars, following the field halo stars, implies that (i) the massive-star IMF is invariant and (ii) well-sampled and the ejecta well-mixed. These place rather stringent requirements on the early star formation. A further conclusion is that the stellar halo could form from any system(s) in which star-formation is short-lived, so that only Type II supernovae have time to enrich the star-forming gas, and enrichment is inefficient so that the mean metallicity is kept low. These could be star clusters, galaxies, or transient structures.

The old age and 'Type II' elemental abundances of the bulk of the field halo stars limits any late merging to be of systems that formed stars early, with short duration; we know of only one such luminous dwarf galaxy with no de- 


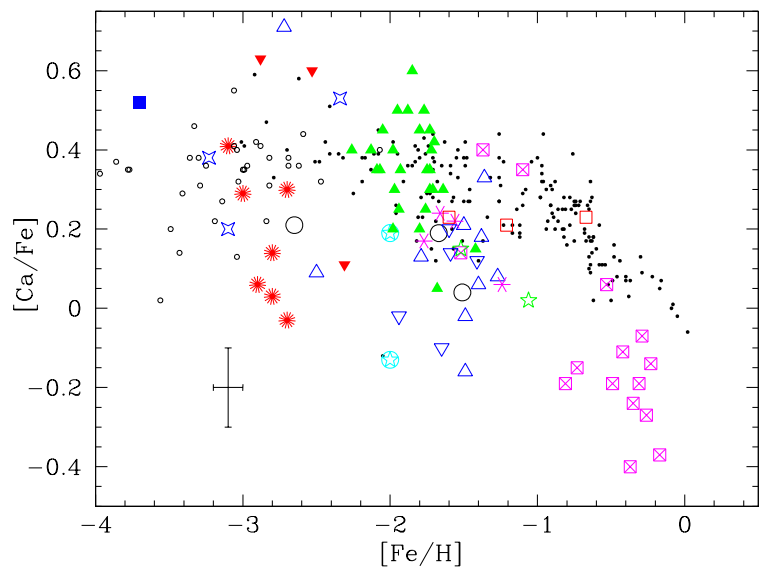

Fig. 4 Abundance of calcium, an $\alpha$-element, relative to iron, for field stars in the Milky Way Galaxy (small open and filled black circles, from Cayrel et al. 2004 and Fulbright 2000, respectively) and stars that are members of representative dwarf spheroidal galaxies, satellites of the Milky Way. Typical errors as indicated in the bottom right. The large black open circles represent stars in the Ursa Minor dSph (Sadakane et al. 2004), the magenta squares, enclosing crosses, represent stars in the Sgr dSph (Monaco et al. 2005), the red many-armed asterisks represent stars in the Sextans dSph (Aoki et al. 2009), the blue open triangles represent stars in the Carina dSph (usual orientation, Koch et al. 2008a; upside down, Shetrone et al. 2003), the green filled triangles represent stars in the Leo II dSph (Shetrone et al. 2009), the open red squares represent stars in the Fornax dSph (Shetrone et al. 2003), the six-armed magenta asterisks represent stars in the Sculptor dSph (Shetrone et al. 2003), the two green open star symbols represent stars in the Leo I dSph (Shetrone et al. 2003), the two cyan circles, enclosing star symbols, represent two stars in the Hercules dSph (Koch et al. 2008b), the blue 4-pointed star symbols represent stars in the Ursa Major I dSph (Frebel et al. 2009), the red upside-down filled triangles represent stars in the Coma Berenices dSph (Frebel et al. 2009) and the filled blue square represents a star in the Boötes I dSph (Norris et al. 2010). These last four $\mathrm{dSph}$ were all discovered within the SDSS imaging dataset and are very faint, fainter than absolute magnitude $M_{V} \sim-7$, well into the regime of globular star clusters (Belokurov et al. 2006, 2007b; Zucker et al. 2006).

tectable age spread, that in Ursa Minor. Even for this system at least one star shows lower ratios of calcium to iron than does the bulk of the stellar halo, as shown in Fig. 4(large black open circles; Sadakane et al. 2004). Recent models within the framework of $\Lambda \mathrm{CDM}$ argue that the field halo of the Milky Way formed from a few LMC-mass systems that were accreted early, and, unlike the LMC, had starformation truncated abruptly at that time (e.g. Robertson et al. 2005). This has not yet been demonstrated within a self-consistent model of star formation and chemical enrichment.

The stars in the Milky Way in Fig. 4 are not identified by kinematics or spatial location into distinct stellar components. When this is done, further insight can be achieved. Fig. 5 shows the results from a large (by the standards of high-resolution spectroscopy) survey of metal-poor stars, with an emphasis on candidate disk members (Ruchti et al. 2010). The sample was selected from the Radial Velocity (RAVE) spectroscopic survey (Steinmetz et al. 2006) as having space motions more consistent with disk than halo, but being of low metallicity; the stellar parameters from the RAVE pipeline were used in this sample selection. The RAVE survey is obtaining moderate-resolution $(\mathcal{R} \sim 8000)$ spectra, around the IR Calcium triplet, for a magnitudelimited sample of bright stars, $I<12$, using the multiobject fibre spectrograph (the $6 \mathrm{dF}$ instrument) on the UK Schmidt telescope. The aim is for a final sample of 1 million stars, allowing for a statistically significant sampling of the kinematic and metallicity distributions of each of the stellar components of the Milky Way. These stars are bright enough that follow-up echelle spectra of 'interesting' subsamples (such as metal-poor disk stars) can be fairly easily obtained on $4 \mathrm{~m}$ - or $8 \mathrm{~m}$-class telescopes, while still probing distances of several kiloparsec from the solar neighbourhood (using giant stars).

The stars with elemental abundances shown in Fig. 5 have been re-classified into halo, thick disk and thin disk on the basis of the stellar parameters derived from the echelle observations, together with isochrone fitting for improved estimates of the gravity (particularly important for the distance estimates for low gravity, luminous red giant stars; we estimate that our distances for giants are accurate to 20-30\%). The stars in the RAVE catalogue are sufficiently bright that proper-motion measurements are available, allowing full space motions to be derived once distance are estimated (the RAVE radial velocities are accurate to better than a few $\mathrm{km} / \mathrm{s}$ ). The component assignment is probabilistic by necessity, since the components have overlapping properties - and indeed characterising, then understanding, that overlap is a major science goal of the full RAVE survey. The different population assignments are indicated by different symbols in the Figure (see Ruchti et al. 2010 for details of the range of criteria used in the assignments). The elemental abundances were derived following the formalism of Fulbright (2000).

It is evident that the thick disk extends at least to $[\mathrm{Fe} / \mathrm{H}]$ $\sim-1.75 \mathrm{dex}$, and that these stars show the same enhanced values of $[\alpha / \mathrm{Fe}]$ as do halo stars, for a range of $\alpha$-elements. Most of the stars classified as thick disk or halo are giants, and probe distances of several kiloparsec from the Sun, the first time that elemental abundances for the metal-poor thick disk has been measured at these distances. Similar elemental abundance results for smaller samples of (high-velocity) thick disk and halo (dwarf) stars at $[\mathrm{Fe} / \mathrm{H}] \sim-1$ dex were reported previously (Nissen \& Schuster 1997), and also for 


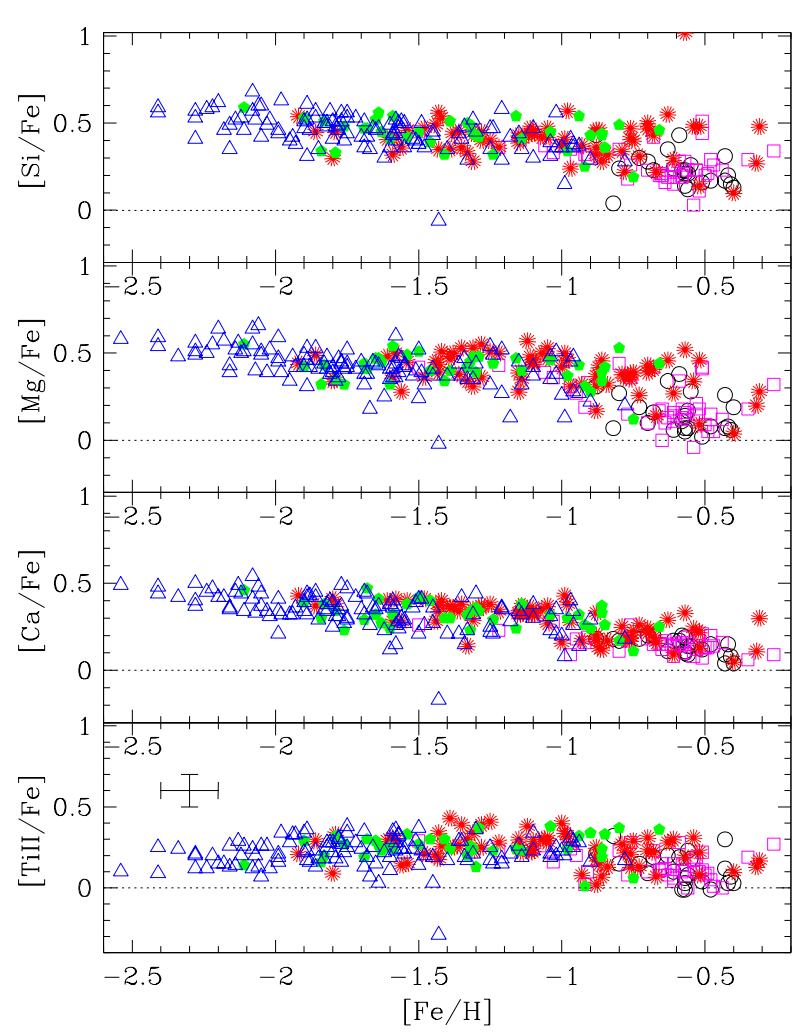

Fig. 5 Elemental abundances, from analyses of highresolution spectra, for stars selected from the RAVE survey database to be low metallicity but with disk-like kinematics. The distances and kinematics have been re-calculated based on the stellar parameters derived from the echelle spectra and isochrone fits. The blue triangles represent stars that have high probability to be halo stars, the red asterisks high probability thick-disk stars, with the green pentagons having similar probability of belonging to either the halo or thick disk. The black circles represent high probability thindisk stars and the magenta open squares are either thin- or thick-disk stars.

a local sample (distances less than $150 \mathrm{pc}$ ) of dwarf stars for the full range in $[\mathrm{Fe} / \mathrm{H}]$ of (Reddy \& Lambert 2008; Reddy, Lambert \& Allende-Prieto 2006). The enhanced values of $[\alpha / \mathrm{Fe}]$ imply that core-collapse supernovae dominated the enrichment of the gas clouds from which these stars formed, which in turn implies that the stars formed within a short time after the onset of star formation. It could still be possible to incorporate an age range greater than $\sim 1 \mathrm{Gyr}$, should this be established by other means, if the stars were to form in distinct sub-units which each had a short duration of star formation, but different times of onset, but this requires some fine-tuning (see Gilmore \& Wyse 1998). A simpler interpretation is that there is a narrow age range and that the metal-poor thick disk (and halo) stars formed during a short-lived epoch of star formation.
The similarity of the elemental abundances across the halo/thick disk transition implies that the core-collapse supernovae that pre-enriched the stars came from a very similar massive-star Initial Mass Function. Further, the low scatter implies good sampling of the massive-star IMF and good mixing within the interstellar medium. This would seem to imply large star-forming regions.

Several models for the thick disk invoke a minor merger and associated heating of a pre-existing thin disk; one then expects to find stars above the disk plane that came from the previous thin disk, together with stars from the nowshredded satellite that merged (e.g. Gilmore, Wyse \& Norris 2002). Distinguishing these two contributions relies upon stellar population signatures. It is clear that the bulk of the thick-disk stars near the solar neighbourhood, for which the mean metallicity is relatively high, $[\mathrm{Fe} / \mathrm{H}] \sim-0.6$ dex, and the mean age old, $\sim 10-12 \mathrm{Gyr}$, must have formed within a fairly deep potential well, since even the Large Magellanic Clouds did not self-enrich to this level until a few Gyr ago. The metal-poor end of the thick disk could more plausibly be associated with debris from accreted dwarf galaxies. The elemental abundance pattern seen in Fig. 51requires that star formation in any such progenitor be very short-lived, which, compared to the typical extended star formation history, and early onset, in surviving dwarf galaxies implies that any such dwarf galaxy be accreted a long time ago. This is consistent with inferences from the old age of stars in the bulk of the thick disk, but not easy to reconcile with the late merging and accretion of satellites predicted in $\Lambda \mathrm{CDM}$ (e.g. Abadi et al. 2003).

Radial migration of stars and gas in disks, due to resonance with transient spiral arms (Sellwood \& Binney 2002), perhaps in concert with the bar (Minchev \& Famaey 2009) is potentially very important. Extremely efficient migration, with a probability that is independent of the amplitude of random motions of the stars, can create a metal-rich thick disk locally out of the inner regions of the disk (Schönrich \& Binney 2009). However, we currently lack an understanding of the efficiency of migration, and this could be tested by large surveys of elemental abundances.

\section{Concluding remarks}

I have only two 'take-home' points:

- Spectroscopy is critical in determining the astrophysics of stellar populations

- Large (planned) imaging surveys need matched spectroscopic capabilities

Acknowledgements. I thank the organisers for their invitation to this stimulating conference. I acknowledge support from the US National Science Foundation (AST-0908326). 


\section{References}

Aaronson, M.: 1983, ApJ 266, L11

Abadi, M., Navarro, J., Steinmetz, M., Eke, V.: 2003, ApJ 597, 21

Aoki, W., Arimoto, N., Sadakane, K. et al.: 2009, A\&A 502, 569

Bell, E., Zucker, D.B., Belokurov, V., et al.: 2008, ApJ 680, 295

Belokurov, V., Zucker, D.B., Evans, N.W., et al.: 2006, ApJ 647, L111

Belokurov, V., Evans, N.W., Bell, E. et al.: 2007a, ApJ 657, L89

Belokurov, V., Zucker, D.B., Evans, N.W., et al.: 2007b, ApJ 654, 897

Belokurov, V., Walker, M., Evans, N.W., et al.: 2010, ApJ 712, L103

Bienaymé, O., Soubiran, C., Mishenina, T., Kovtyukh, V., Siebert, A.: 2006, A\&A 446, 933

Binney, J., Mamon, G.: 1982, MNRAS 200, 361

Cayrel, R., Depagne, E., Spite, M., et al.: 2004, A\&A 416, 1117

Ceverino, D., Dekel, A., Bournaud, F.: 2010, MNRAS submitted (arXiv:0907.3271)

Chapman, S., Ibata, R., Irwin, M., et al.: 2008, MNRAS 390, 1437

Chou, M.-Y., Cunha, K., Majewski, S.R., et al.: 2010, ApJ 708, 1290

Durrell, P., Harris, W., Pritchet, C... 2004, AJ 128, 260

Elmegreen, B.G., Elmegreen, D.M.: 2006, ApJ 650, 644

Fakhouri, O., Ma, C.-P., Boylan-Kolchin, M.: 2010, MNRAS submitted (arXiv:1001.2304)

Fardal, M.A., Babul, A., Guhathakurta, P., Gilbert, K.M., Dodge, C.: 2008, ApJ 682, L33

Fellhauer, M., Belokurov, V., Evans, N.W., et al.: 2006, ApJ 651, 167

Ferguson, Annette M. N.; Irwin, Michael J.; Ibata, R., et al.: 2002, AJ 124,1452

Font, A., Johnston, K., Ferguson, A., et al.: 2008, ApJ 673, 215

Frebel, A., Simon, J., Geha, M., Willman, B.: 2010, ApJ, 708, 560

Fulbright, J.: 2000, AJ 120, 1841

Genzel, R.: 2009, in 'The Galaxy Disk in Cosmological Context', IAU Symposium 254, eds J. Andersen, J. Bland-Hawthorn, \& B. Nordström, Cambridge, CUP, p. 33

Gerhard, O.: 1993, MNRAS 265, 213

Gerhard, O., Jeske, G., Saglia, R., Bender, R.: 1998, MNRAS 295, 197

Gibson, B.K.: 1998, ApJ 501, 675

Gilbert, K., Guhathakurta, P., Kollipara, P., et al.: 2009, ApJ 705, 1275

Gilmore, G., Wyse, R.F.G.: 1998, AJ 116, 748

Gilmore, G., Wyse, R.F.G., Norris, J.E.: 2002, ApJ 574, L39

Gilmore, G., Wilkinson, M., Wyse, R.F.G., et al.: 2007, ApJ 663, 948

Governato, F., Brook, C.B., Brooks, A.M., et al.: 2009, MNRAS 398,312

Grcevich, J., Putman, M.E.: 2009, ApJ 696, 385

Holmberg, J., Flynn, C.: 2004, MNRAS 352, 440

Huang, S., Carlberg, R.G.: 1997, ApJ 480, 503

Ibata, R.A., Gilmore, G., Irwin, M.J.: 1994, Nat 370, 194

Ibata, R.A., Gilmore, G.: 1995, MNRAS 275, 591

Ibata, R.A., Chapman, S., Ferguson, A.M.N., et al.: 2004, MNRAS 351,117

Ibata, R.A., Irwin, M., Lewis, G., et al.: 2001, Nat 412, 49

Ibata, R.A., Wyse, R.F.G., Gilmore, G., Irwin, M.J., Suntzeff, N.: 1997, AJ 113, 634

Johnston, K.V., Bullock, J.S., Sharma, S., et al.: 2008, ApJ 689, 936

Kalirai, J., Beaton, R., Geha, M., et al.: 2010, ApJ 711, 671
Kazantzidis, S., Zentner, A.R., Kravtsov, A.V., et al.: 2009, ApJ 700,1896

Klypin, A., Kravtsov, A.V., Valenzuela, O., Prada, F.: 1999, ApJ 522,82

Kobayashi, C., Umeda, H., Nomoto, K., Tominaga, N., Ohkubo, T.: 2006, ApJ 653, 1145

Koch, A., Grebel, A., Gilmore, G., et al.: 2008a, AJ 135, 1580

Koch, A., McWilliam, A., Grebel, E., et al.: 2008b, ApJ 668, L13

Khochfar, S., Burkert, A.: 2006, A\&A 445, 403

Komatsu, E., Dunkley, J., Nolta, M.R., et al. (WMAP team): 2009, ApJS 180, 330

Kuijken, K., Gilmore, G.: 1989, MNRAS 239, 605

Kuijken, K., Gilmore, G.: 1991, ApJ 367, L9

Kusenko, A.: 2009, Phys Rep 481, 1

Layden, A.C., Sarajedini, A.: 2000, AJ 119, 1760

Letarte, B., Chapman, S., Collins, M., et al.: 2009, MNRAS, 400, 1472

McConnachie, A., Irwin, M.: 2006, MNRAS 365, 1263

McConnachie, A., Irwin, M., Ibata, R. et al.: 2009, Nat 461, 66

McWilliam, A., Smecker-Hane, T.A.: 2005, ApJ 622, L29

Martin, N., McConnachie, A., Irwin, M., et al.: 2009, ApJ 705, 758

Martin, N., de Jong, J., Rix, H.-W.: 2008, ApJ 684, 1075

Matteucci, F., Spitoni, E., Recchi, S., Valiante, R.: 2009, A\&A 501,531

Mihos, J.C., Hernquist, L.: 1996, ApJ 464, 641

Minchev, I., Famaey, B.: 2009, ApJ submitted (arXiv:0911.1794)

Monaco, L., Bellazzini, M., Bonifacio, P., et al.: 2005, A\&A 441, 141

Moore, B., Ghigna, S., Governato, F., et al.: 1999, ApJ 524, L19

Mould, J., Kristian, J.: 1986, ApJ 305, 591

Navarro, J., Frenk, C., White, S.D.M.: 1996, ApJ 462, 563

Nissen, P., Schuster, W.: 1997, A\&A 326, 751

Norris, J.E., Yong, D., Gilmore, G., Wyse, R.F.G.: 2010, ApJ 711, L350

Ostriker, J.P., Steinhardt, P.: 2003, Sci 300, 1909

Peebles, P.J.E., Nusser, A.: 2010, arXiv:1001.1484

Percival, W., Reid, B.A., Eisenstein, D.J., et al.: 2010, MNRAS 401, 2148

Prior, S., Da Costa, G., Keller, S.: 2009, ApJ 704, 1327

Putman, M., Peek, J., Muratov, A., et al.: 2009, ApJ 703, 1486

Quinn, P., Goodman, J.:1986, ApJ 309, 472

Read, J.I., Mayer, L., Brooks, A.M., Governato, F., Lake, G.: 2009, MNRAS 397, 44

Reddy, B., Lambert, D.: 2008, MNRAS 391, 95

Reddy, B., Lambert, D., Allende Prieto, C.: 2006, MNRAS 367, 1329

Robertson, Brant; Bullock, James S.; Font, Andreea S.; Johnston, Kathryn V.; Hernquist, L.: 2005, ApJ 632, 872

Ruchti, G., Fulbright, J., Wyse, R.F.G., et al.: 2010, in preparation

Sadakane, K., Arimoto, N., Ikuta, C., et al.: 2004, PASJ 56, 1041

Sbordone, L., Bonifacio, P., Buonanno, R., Marconi, G., Monaco, L., Zaggia, S.: 2007 A\&A, 465, 815

Scannapieco, C., White, S.D.M., Springel, V., Tissera, P.B.: 2009, MNRAS 396, 696

Schönrich, R., Binney, J.: 2009, MNRAS 399, 1145

Sellwood, J., Binney, J.: 2002, MNRAS 336, 785

Shetrone, M., Venn, K., Tolstoy, E., et al. : 2003, AJ 125, 684

Shetrone, M., Siegel, M.H., Cook, D., Bosler, T.: 2009, AJ 137, 62

Siegel, M.H., Dotter, A., Majewski, S.R., et al.: 2007, ApJ 667, L57

Stadel, J., Potter, D., Moore, B., et al.: 2009, MNRAS 398, L21

Steinmetz, M.,Zwitter, T., Siebert, A., et al. (the RAVE collaboration): 2006, AJ 132, 1645 
Stewart, K.R., Bullock, J. S., Wechsler, R.H., et al.: 2008, ApJ 683, 597

Tanaka, M., Chiba, M., Komiyama, Y., Guhathakurta, P., Kalirai, J., Iye, M.: 2010, ApJ 708, 1168

Tinsley, B.M., Larson, R.B.: 1979, MNRAS 186, 503

Toth, G., Ostriker, J.P.: 1992, ApJ 389, 5

Unavane, M., Wyse, R.F.G. \& Gilmore, G.: 1996, MNRAS 278, 727

Venn, K., Irwin, M., Shetrone, M., et al.: 2004, AJ 128, 1177

Walker, M., Mateo, M., Olszewski, E., et al.: 2009, ApJ 704, 1274

Watkins, L. L.; Evans, N. W.; Belokurov, V., et al.: 2009, MNRAS 398, 1757

White, S.D.M., Rees, M.J.: 1978, MNRAS 183, 341

Wyse, R.F.G.: 2001, in 'Galaxy Disks and Disk Galaxies', ASP Conference Series vol. 230, eds J. Funes \& E. Corsini. San Francisco, ASP, p. 71

Wyse, R.F.G., Gilmore, G.: 1988, AJ 95, 1404

Wyse, R.F.G., Gilmore, G.: 1993, in 'The Globular Cluster-Galaxy Connection', ASP Conference Series vol. 48, eds G. Smith \& J. Brodie. San Francisco, ASP, p. 727

Wyse, R.F.G., Gilmore, G., Franx, M.: 1997, ARAA 35, 637

Zolotov, A., Willman, B., Brooks, A.M., et al.: 2009, ApJ 702, 1058

Zucker, D.B., Belokurov, V., Evans, N.W., et al.: 2006, ApJ 650, L41

Zurek, W.H., Quinn, P.J., Salmon, J.K.: 1988, ApJ 330, 519 